BULL. AUSTRAL. MATH. SOC.

\title{
ABSTRACT BOCHINER-WEIL-RAIKOV THEOREM IN TOPOLOGICAL ALGEBRAS
}

\author{
Maria Fragoulopoulou
}

\begin{abstract}
Each continuous positive linear form on a commutative locally m-convex *algebra $E$ with a bounded approximate identity, accepts an integral representation on the hermitian spectrum (hermitian characters) of $E$. An alternative form of the latter is also obtained. The presented results constitute an abstract form of the Bochner-Weil-Raikov theorem within the frame of topological *-algebras.
\end{abstract}

In the context of commutative Banach *algebras theory, a "Bochner type" theorem yields, for positive and extendable linear functionals, integral representations on the Gel'fand spaces (maximal ideal spaces) of the Banach algebras considered ( $c f$. for instance, [3, p. 97, Theorem 26.I]). Lumer introduces in [4] a new, simpler and more general approach for obtaining the preceding result, replacing the continuous involution of the given Banach algebra $E$ by an appropriate finite group of transformations of $E$. Following Lumer's lines of thought we obtained, in [1], integral representations for continuous linear forms on commutative locally m-convex algebras (cf. [1, Theorems 3.6, 4.6]), getting thus, among other things, a Bochner-Weil-Raikov theorem in the non-normed topological algebra case [1, Corollary 3.8]. Now, using the technique developed in [1], as well as results from the general representation theory of topological *-algebras [2], we give in this note the Banach *-algebras analogue in our case (Theorem 2.1 and/or Theorem 2.2) of the so-called

Received 9 March 1982. 
"abstract form of Bocher's theorem" ( $c f$. for example, [8, Theorem 9.14] and/or [9, Theorem 11.32]).

1.

A locally m-convex *-algebra is a topological algebra $E$ with an involution *, whose topology is defined by a family $\left(p_{\alpha}\right)_{\alpha \in A}$ of *-preserving submultiplicative semi-norms. The "*-preserving property" evidently implies the continuity of * and it is of course a stronger condition than that of the continuity itself. However there is no loss of generality by assuming it, since having a continuous involution one always defines a new family of *-preserving submultiplicative semi-norms, equivalent to the original one.

Let us now recall some definitions and notation from [1] which we shall use in the sequel. If $E$ is a locally m-convex algebra and $\Gamma$ a finite group of additive multiplicative transformations of $E$ such that $p_{\alpha}(\gamma(x))=p_{\alpha}(x)$ for any $\gamma \in \Gamma, \alpha \in A, x \in E$, then the pair (E, $\left.\Gamma\right)$ is called a $\Gamma$-Lumer system. Thus, if $\left(E_{\alpha} \equiv E / \operatorname{ker}\left(p_{\alpha}\right)\right),\left(\tilde{E}_{\alpha}\right.$ (completion of $\left.E_{\alpha}\right)$ ) are the projective systems of normed respectively Banach *-algebras corresponding to $E[7]$, then each $\left(\tilde{E}_{\alpha}, \tilde{\Gamma}_{\alpha}\right)$ is a $\tilde{\Gamma}_{\alpha}$-Lumer system for $\tilde{E}_{\alpha}$, where $\tilde{Y}_{\alpha} \in \tilde{\Gamma}_{\alpha}$ is the continuous extension of $\gamma_{\alpha}: E_{\alpha} \rightarrow E_{\alpha}: x_{\alpha}\left(\equiv x+\operatorname{ker}\left(p_{\alpha}\right)\right) \mapsto \gamma_{\alpha}\left(x_{\alpha}\right)=\gamma(x)$, with $\gamma \in \Gamma, \alpha \in A$ (cf. [1, Lemma 3.2]). In this respect, an element $f \in E^{\prime}$ (topological dual of $E$ ) is said to satisfy condition ( () if there is $\alpha \in A$ and $\tau>0$ such that

$$
\text { (1.1) } \quad\left|f_{\alpha}(z)\right| \leq 2 \cdot N_{\alpha}(z), z \in \tilde{E}_{\alpha} \text {, }
$$

for every $\tilde{\Gamma}_{\alpha}$-invariant norm $N_{\alpha}$ on $\tilde{E}_{\alpha}$ equivalent to the given one, where $f_{\alpha} \in\left(\tilde{E}_{\alpha}\right)^{\prime}: f_{\alpha}\left(x_{\alpha}\right)=f(x), x_{\alpha} \in E_{\alpha}$. The positive number $\tau$ is the same for all $N_{\alpha}$, whereas $l=1=f(e)$ when $E$ has an identity $e$ and $f$ is a continuous positive linear form on $E$ preserving $e$. The index $\alpha \in A$ in (1.1) is that provided by the continuity of $f$.

In the sequel, if $E$ is a commutative locally m-convex algebra, 
$M(E)$ will denote the spectrum (maximal ideal space) of $E$ and $C_{C}(M(E)$ ) the algebra of the complex-valued continuous functions on $M(E)$ with the topology " $c$ " of compact convergence. Thus $E^{\wedge} \subseteq C_{c}(M(E))$ will stand for the Gel'fand transform algebra of $E$.

Concerning the above, one has the next integral representation theorem.

THEOREM 1.1 (cf. [1, Theorem 3.6]). Let $E$ be a commutative locally m-convex algebra with identity and continuous Gel'fand map. Let also $(E, \Gamma)$ be a $\Gamma$-Lumer system for $E$ and $f \in E^{\prime}$. Then $f$ satisfies condition (l) if and only if $f(x)=\mu(\hat{x}), x \in E$, with $\mu$ a (complex) measure on $M(E)$ of positive total variation at most $Z$.

When $l=f\left(l_{E}\right)=1$, we get Lumer's theorem $[4, \S 1]$ for unital Banach algebras. On the other hand, the continuity of the Gel'fand map in Theorem 1.1 is only used for the proof of the "if" part; thus it is not needed for the proof of the Bochner-Weil-Raikov theorem which is a consequence of the foregoing one. That is, one has

COROLLARY 1.1 (cf. [1, Corollary 3.8]). Let $E$ be a commutative locally m-convex *-algebra such that $\left(x^{*}\right)^{\wedge}=(\hat{x})^{-}, x \in E$. Let also $f \in E^{\prime}$. Then $f$ is positive and extendable (onto the respective unital algebra $E_{1}$ of $E$ ) if and only if $f(x)=\mu(\hat{x}), x \in E$, with $\mu$ a (complex) positive finite measure on $M(E)$.

The respective unital algebra $E_{1}$ of a locally m-convex *algebra $E$ is an algebra of the kind of $E$, whose involution is defined by $(x, \lambda)^{*}=\left(x^{*}, \bar{\lambda}\right),(x, \lambda) \equiv x_{1} \in E_{\mathcal{I}}$, and its topology by the family $\left(p_{1, \alpha}\right)$ of submultiplicative seminorms, with $p_{1, \alpha}(x, \lambda)=p_{\alpha}(x)+|\lambda|$, $(x, \lambda) \in E_{1}, \alpha \in A$.

\section{2.}

Let now $E$ be a locally m-convex *-algebra with bounded approximate identity, that is, a net $\left(e_{i}\right)_{i \in I}$ in $E$ such that $\lim _{i}\left(e_{i} x\right)=x=\lim _{i}\left(x e_{i}\right), x \in E$ and $p_{\alpha}\left(e_{i}\right) \leq 1$, for any $i \in I$, 
$\alpha \in A$. Then, if $P(E)$ denotes the set of all continuous positive linear forms on $E$, one has that each $f \in P(E)$ is extendable (cf. [2, Lemma 3.3]). On the other hand, restricting ourselves on the hermitian spectmum $D^{*}(E) \quad\left(\equiv\left\{h \in M(E): h\left(x^{*}\right)=\overline{h(x)}, x \in E\right\}\right)$ of $E$, we clearly have $\left(x^{*}\right)^{\wedge}=(\hat{x})^{-}$for every $x \in E$. In this concern, one gets the following abstract form of the Bochner-Weil-Raikov theorem, whose proof, after the preceding discussion can be obtained as a consequence of Corollary 1.1 . However, for completeness sake, we present still a modified proof.

THEOREM 2.1. Let $E$ be a commutative Zocally m-convex *-algebra with a bounded approximate identity and $f \in E^{\prime}$. Then $f$ is positive if and only if $f(x)=\mu(\hat{x}), x \in E$, with $\mu$ a positive finite measure on $D^{*}(E)$ (of total variation less than or equal to 1 ).

Proof. Let $E_{1}$ be the respective unital algebra of $E$. Then involution on $E_{1}$ defines a finite group $\Gamma_{1}$ of transformations of $E_{1}$ with $\Gamma_{1}=\left\{\gamma_{1}, \gamma_{1}^{-1}, i d_{E_{1}}\right\}$ and $\gamma_{1}\left(x_{1}\right)=x_{1}^{*}, x_{1} \in E_{1}$, in such a way that $\left(E_{1}, \Gamma_{1}\right)$ is a $\Gamma_{1}$-Lumer system for $E_{1}$. On the other hand, since $f \in P(E)$, it is extended to an element $f_{1}$ of $P\left(E_{1}\right)$ with $f_{1}(0,1)=\left\|f_{\alpha}\right\| \leq 1$, where $f_{\alpha} \in P\left(\tilde{E}_{\alpha}^{\prime}\right)$ with $f_{\alpha}\left(x_{\alpha}\right)=f(x), x_{\alpha} \in E_{\alpha}$ (cf. [2, Proposition 3.4 and Theorem 3.1]) and $\alpha$ that index in $A$ provided by the continuity of $f$. Now, for the same index $\alpha, f_{1}$ defines $f_{1, \alpha} \in P\left(\tilde{E}_{1, \alpha}\right)\left(E_{1, \alpha} \equiv E_{1} / \operatorname{ker}\left(p_{1, \alpha}\right)\right)$ in the preceding way [2, Theorem 3.1], so that ([9, Theorem $11.31(d)]$ ),

$$
\left|f_{1, \alpha}\left(\omega_{1, \alpha}\right)\right| \leq\left\|f_{\alpha}\right\| r_{\alpha}\left(\omega_{1, \alpha}\right) \leq\left\|f_{\alpha}\right\| N_{1, \alpha}\left(\omega_{1, \alpha}\right),
$$

for every self-adjoint element $\omega_{1, \alpha} \in \tilde{E}_{1, \alpha}$ and every $\tilde{\Gamma}_{1, \alpha}$ invariant norm $N_{1, \alpha}$ on $\tilde{E}_{1, \alpha}$ equivalent to the original one $\tilde{\dot{p}}_{1, \alpha}$ $\left(\dot{p}_{1, \alpha}\left(x_{1, \alpha}\right) \equiv p_{1, \alpha}\left(x_{1}\right), x_{1, \alpha} \in E_{1, \alpha}\right)$, where $r_{\alpha}$ is the spectral radius of $\omega_{1, \alpha}$ in $\tilde{E}_{1, \alpha}$. Now, since each element $z \in \tilde{E}_{1, \alpha}$ is of the form $s+i t$, with $s, t$ self-adjoint elements in $\tilde{E}_{1, \alpha}$, one finally gets $\left|f_{1, \alpha}(z)\right| \leq\left\|f_{\alpha}\right\| N_{1, \alpha}(z)$, for every $z \in \tilde{E}_{1, \alpha}$ and every $N_{1, \alpha}$ as before 
(cf. also [4, $\$ 2$, Proof of Corollary]); that is $f_{1}$ satisfies condition ( $Z$ ) with $Z=\left\|f_{\alpha}\right\|$, hence the assertion follows by Theorem 1.1 (cf. also discussion after it) restricting $\mu$ on $D^{*}(E)$. The "if" part is a consequence of $\left(x^{*}\right)^{\wedge}=(x)^{-}, x \in E$.

For the Banach *-algebras analogue of Theorem 2.1, see [8, Theorem 9.14] as well as [9, Theorem 11.32].

COROLLARY 2.1. Let $G$ be a locally compact abelian group and $E$ a commutative complete locally m-convex *algebra with a bounded approximate identity. Let also $L_{E}^{1}(G)$ be the generalized group algebra of $G$ [5] and $f \in\left(L_{E}^{I}(G)\right)^{\prime}$. Then $f$ is positive if and only if $f(\vec{\varphi})=\mu(\vec{\varphi}), \vec{\varphi} \in L_{E}^{\perp}(G)$, where $\mu$ is a positive finite measure on $D *\left(L_{E}^{1}(G)\right)$.

Proof. $L_{E}^{I}(G)=L^{1}(G) \underset{\pi}{\hat{\otimes}} E \quad[5, p .288$, Lemma $]$ is a commutative complete locally m-convex *-algebra with a bounded approximate identity (cf. for the latter [1, Corollary 5.5]) so that the assertion follows by the preceding theorem.

Regarding the supposition of the continuity of $f$ in Theorem 2.1, one could dispense with it at the cost, however, of the continuity of the Gel'fand map of the topological algebra involved. Thus, we have the following alternative of Theorem 2.1.

THEOREM 2.2. Let $E$ be a commutative locally m-convex *algebra with a bounded approximate identity and continuous Gel'fand map. Then $f \in P(E)$ if and only if $f(x)=\mu(\hat{x}), x \in E$, with $\mu$ a positive finite measure on $D^{*}(E)$ (of total variation less than or equal to 1 ).

Proof. We work out only the continuity of $f$ in the "if" part. Let $\mu_{1}$ be a positive finite measure on $M\left(E_{1}\right)$ and $\mu$ its restriction to $D^{*}(E)$ such that $f(x)=\mu(\hat{x}), x \in E$. The continuity of $\mu_{1}$ implies the existence of a compact subset $K$ of $M\left(E_{1}\right)$ such that $\left|\mu_{1}\left(\hat{x}_{1}\right)\right| \leq q_{K}\left(\hat{x}_{1}\right), x_{1} \in E_{1}$, where $q_{K}\left(\hat{x}_{1}\right)=\sup \left\{\left|h_{1}\left(x_{1}\right)\right|: h_{1} \in K\right\}$. 
Now continuity of the Gel'fand map of $E$ implies that of $E_{1}$ [6], hence [6, Theorem III, 4.I] there is $\alpha \in A$ with $K \subseteq M\left(\tilde{E}_{1, \alpha}\right)$, so that $\left|\mu_{1}\left(\hat{x}_{1}\right)\right| \leq q_{M\left(\tilde{E}_{1, \alpha}\right)}\left(\hat{x}_{1}\right)=r_{\alpha}\left(x_{1, \alpha}\right) \leq \dot{p}_{1, \alpha}\left(x_{1, \alpha}\right) \equiv p_{1, \alpha}\left(x_{1}\right), x_{1} \in E_{1}$. Thus $|f(x)|=|\mu(\hat{x})| \leq p_{\alpha}(x)$ for every $x \in E$ and some $\alpha \in A$.

\section{References}

[1] Maria Fragoulopoulou, "Integral representations of linear forms on topological algebras", Comment. Math. Prace Mat. 21 (1980), 43-53.

[2] Maria Fragoulopoulou, "Spaces of representations and enveloping l.m.c. *-algebras", Pacific J. Math. 95 (1981), 61-73.

[3] Lynn H. Loomis, An introduction to abstract harmonic analysis (Van Nostrand, Toronto, New York, London, 1953).

[4] G. Lumer, "Bochner's theorem, states, and the Fourier transforms of measures", Studia Math. 46 (1973), 135-140.

[5] Anastasios Mallios, "Note on the tensor products and harmonic analysis", Math. Ann. 173 (1967), 287-289.

[6] Anastasios Mallios, General theory of topological algebras: selected topics (in preparation).

[7] Ernest A. Michael, Locally multiplicatively-convex topological algebras (Memoirs of the American Mathematical Society, 11. American Mathematical Society, Providence, Rhode Island, 1952).

[8] Richard D. Mosak, Banach algebras (University of Chicago Press, Chicago, London, 1975).

[9] Walter Rudin, Functional analysis (McGraw-Hill, New York, London, Sydney, 1973).

Mathematical Institute,

University of Athens,

57 Solonos Street,

Athens 143, Greece. 